\title{
Trabajadores en contacto con amianto: pautas de seguimiento
}

Workers in contact with asbestos: follow-up patterns

\section{Pascal ${ }^{1}$, B. Marín Martínez ${ }^{2}$}

\section{RESUMEN}

La exposición a amianto se asocia al desarrollo de patología pleuropulmonar benigna y maligna. Existe una amplia regulación en los últimos años, desde el establecimiento de condiciones en los trabajos con exposición a amianto hasta las sucesivas prohibiciones sobre su utilización. Para detectar estas patologías se han diseñado unos reconocimientos médicos periódicos en los trabajadores expuestos y teniendo en cuenta el largo período de latencia también deben realizarse a trabajadores jubilados expuestos o postocupacionales.

Palabras clave. Amianto.

\begin{abstract}
Exposure to asbestos is associated with the development of benign and malign pleuropulmonary pathology. In recent years a broad set of regulations has been set in place, from the establishment of conditions in work involving exposure to asbestos to successive prohibitions on its use. Periodical medical checkups have been designed o detect these pathologies in exposed workers and, considering the long period of latency, they should also be carried out on retired workers or on those who in the past have suffered such exposure.
\end{abstract}

Key words. Asbestos.

An. Sist. Sanit. Navar. 2005; 28 (Supl. 1): 45-49.

1. Sección de Neumología. Hospital Virgen del Camino. Pamplona.

2. Sección de Neumología. Hospital de Navarra. Pamplona.

\author{
Correspondencia: \\ Idoya Pascal Martínez \\ Sección de Neumología \\ Hospital Virgen del Camino \\ Irunlarrea, 4 \\ 31008 Pamplona \\ Tfno. 848429666 \\ e-mail: ipascalm@cfnavarra.es
}




\section{INTRODUCCIÓN}

Se han descrito previamente las enfermedades pleuropulmonares producidas en trabajadores expuestos a amianto, patología benigna como placas pleurales, fibrosis pleural difusa, derrame pleural, atelectasia redonda y asbestosis, así como malignas, mesotelioma y cáncer de pulmón. La toxicidad de las fibras de amianto depende de la duración y de la intensidad de la exposición, existiendo un tiempo de latencia entre ésta y la aparición de la patología.

Desde el Ministerio de Trabajo y Seguridad Social se protege al trabajador frente al ambiente de trabajo, disminuyendo y eliminando la exposición, así como detectando precozmente la enfermedad mediante controles médicos periódicos. Para dicho fin se creó en 1984 el Reglamento sobre trabajos con Riesgo de Amianto ${ }^{1}$, donde se establecieron las medidas mínimas básicas a tener en cuenta en actividades laborales en relación con el amianto para evitar patología derivada del contacto con dicho agente y realización de controles médicos preventivos de los trabajadores. Posteriormente se realizaron modificaciones en dicho documento; en $1993^{2}$, se fijaron normas sobre concentración y dosis acumuladas de amianto en trabajadores potencialmente expuestos. En $2001^{3}$, se prohibió el uso del amianto excepto en dos campos, demolición y fabricación de cloroálcalis.

En el medio laboral, se evalúa el riesgo de amianto mediante la determinación de fibras de amianto en el aire, clasificando a los trabajadores en:
- Trabajadores no potencialmente expuestos, ocupados en actividades con nivel ambiental de fibras de amianto menor que el valor de referencia establecido como de riesgo.

- Trabajadores potencialmente expuestos, en actividades con nivel ambiental de fibras de amianto igual o superior al establecido como de riesgo para producir patología.

Los reconocimientos que se deben realizar al trabajador están recogidos en el Protocolo de Vigilancia Sanitaria específica del Amianto de la Comisión de Salud Pública del Consejo Interterritorial de Salud ${ }^{4}$ (Tabla 1).

Constan de:

- Reconocimiento médico al inicio de la actividad laboral de riesgo.

- Reconocimientos médicos periódicos durante la actividad laboral.

- Reconocimientos médicos postocupacionales, tras el cese de dicha actividad laboral.

\section{RECONOCIMIENTO MÉDICO AL INICIO DE LA ACTIVIDAD LABORAL}

A todo trabajador previamente a la incorporación a un puesto de trabajo en contacto con amianto, se le realizará un reconocimiento que incluya:

- Historia laboral anterior; se interrogará sobre actividades laborales previas específicamente sobre aquellas con riesgo de exposición a amianto. A su vez se evaluará dicho

Tabla 1. Protocolo de exámenes de salud para trabajadores con exposición a amianto.

\begin{tabular}{llccc}
\hline Reconocimiento & Trabajadores & $\begin{array}{l}\text { Anamnesis } \\
\text { Inspección } \\
\text { Auscultación } \\
\text { C. antitabaco }\end{array}$ & $\begin{array}{c}\text { Estudio } \\
\text { radiológico }\end{array}$ & $\begin{array}{c}\text { Pruebas } \\
\text { Funcionales } \\
\text { respiratorias }\end{array}$ \\
\hline $\begin{array}{l}\text { Inicial } \\
\text { Periódico }\end{array}$ & $\begin{array}{c}\text { Sí } \\
\text { Anual }\end{array}$ & $\begin{array}{c}\text { Sí } \\
\text { Anual }\end{array}$ & Anual \\
Potencialmente & $\begin{array}{l}\text { No potencialmente } \\
\text { expuestos } \\
\text { Potencialmente } \\
\text { expuestos } \\
\text { No potencialmente } \\
\text { expuestos }\end{array}$ & Anual & Cada 3 años & Cada 3 años \\
& Anual & Anual & Anual \\
\hline
\end{tabular}


riesgo en el trabajo a realizar en el momento actual.

- Anamnesis haciendo constar el hábito tabáquico, enfermedades respiratorias previas e interrogatorio sintomático sobre características de tos, expectoración y disnea.

- Exploración física.

- Exploraciones complementarias, con RX de tórax en dos proyecciones (PA y lateral) y estudio funcional respiratorio.

- Consejo médico antitabaco; es conocido el efecto carcinógeno del tabaco por sí mismo así como el de las fibras de amianto. Se han realizado estudios que sugieren un aumento del riesgo oncogénico cuando coinciden ambos factores ${ }^{5}$.

Este reconocimiento médico al inicio de la actividad laboral supone un punto de referencia respecto a posteriores valoraciones del trabajador.

- Se considerará no apto para la realización de dicho trabajo aquella persona que presente:

- Alteraciones de las vías aéreas superiores que predispongan la aparición de patología neumoconiótica.

- Neumopatía crónica con alteración clínica o funcional.

- Cardiopatía crónica incapacitante.

\section{RECONOCIMIENTOS MÉDICOS PERIÓDICOS A LO LARGO DE LA ACTIVIDAD LABORAL DE RIESGO}

\section{Trabajadores potencialmente expuestos}

En aquellos trabajadores potencialmente expuestos (con nivel ambiental laboral de fibras de asbesto considerado de riesgo para desencadenar patología), se realizará anualmente la siguiente valoración:

Historia laboral anterior.

Historia clínica, especificando hábito tabáquico y síntomas respiratorios (tos, expectoración y disnea).

Exploración física; inspección, valorando la aparición de acropaquias; y auscultación pulmonar detectando precozmente crepitantes (que sugieren asbestosis) y/o hipoventilación alveolar (derrame pleural, fibrosis pleural, mesotelioma).

Exploraciones complementarias: RX de tórax (PA y lateral) ampliándose el estudio a otras proyecciones (ej. decúbito lateral, ante sospecha de derrame pleural) de considerarse oportuno. Los hallazgos obtenidos se evaluarán según la Clasificación Internacional de la OIT (1980). La TACAR torácica es una exploración radiológica que se solicitará para confirmar los hallazgos de RX de tórax, cuando existan dudas con esta técnica o cuando ante RX de tórax normal exista la sospecha médica de patología relacionada con el amianto, por presentar pruebas funcionales o exploración física que lo sugieran. Sin embargo la TACAR torácica no se considera una exploración rutinaria en estos reconocimientos. Los criterios según SEPAR ${ }^{6}$ para la realización de TACAR en reconocimientos periódicos a trabajadores con exposición a amianto se reflejan en la tabla 2 .

- Pruebas de función respiratoria: espirometría forzada de forma rutinaria con medición de volumen respiratorio forzado en el primer segundo (FEV1), capacidad vital forzada (FVC) y flujo respiratorio de las pequeñas vías (FEF 25-75\%); de

Tabla 2. Criterios para la realización de tomografía computarizada de alta resolución en reconocimientos periódicos a trabajadores con exposición a amianto.

- Sospecha de enfermedad del parénquima pulmonar en la radiografía simple.

- Cambios en el parénquima con respecto a la revisión anterior.

- Aumento del grosor o extensión de placas pleurales o de engrosamientos pleurales.

- Dolor pleural en las placas pleurales previamente asintomáticas.

- Alteraciones en las pruebas de función respiratoria.

- Caída de las pruebas de función respiratoria por encima de lo normal con respecto a reconocimientos previos. 
presentar alteración en los parámetros anteriores se realizará espirometría tras broncodilatación y test de difusión de CO (DLCO).

- Consejo antitabaco.

\section{Trabajadores no potencialmente expuestos}

En trabajadores no potencialmente expuestos, (en ambiente laboral con nivel de fibras de asbesto por debajo del considerado de riesgo para desencadenar patología), se realizará anualmente:

- Historia laboral anterior.

- Historia clínica interrogando sobre sintomatología respiratoria.

- Exploración física con inspección y auscultación pulmonar.

- Consejo antitabaco.

Y cada tres años: RX tórax PA y lateral y estudio funcional respiratorio (espirometría en todos los casos y DLCO según criterio médico).

En cualquiera de los casos anteriores, el trabajador abandonará la actividad laboral por incapacidad temporal y será remitido para estudio neumológico cuando en un reconocimiento se detecte:

- Disnea de esfuerzo.

- Dolor torácico persistente no secundario a otra patología.

- Crepitantes inspiratorios persistentes basales.

- Alteraciones radiológicas pleurales no filiadas o de nueva aparición, o alteraciones sugestivas de patología intersticial difusa.

- Alteraciones de función respiratoria compatibles con patología.

\section{RECONOCIMIENTOS MÉDICOS POSTOCUPACIONALES}

Toda persona con antecedente de exposición laboral a amianto realizará controles periódicos tras finalizar su actividad de riesgo, dado el tiempo de latencia que existe desde exposición a dicho material y su patología asociada.
En aquellos que estuvieron expuestos potencialmente a niveles de riesgo, se realizará anualmente:

- Historia laboral.

- Historia clínica: sintomatología respiratoria.

- Exploración física con inspección y auscultación pulmonar.

- RX de tórax: PA y lateral.

- Estudio funcional respiratorio (espirometría forzada y DLCO según criterio médico).

- Consejo antitabaco.

En aquellos no expuestos potencialmente a niveles de amianto de riesgo, se realizará anualmente:

- Historia laboral.

- Historia clínica: sintomatología respiratoria.

- Exploración física con inspección y auscultación pulmonar.

- Estudio funcional respiratorio (espirometría forzada y DLCO según criterio médico).

- Consejo antitabaco.

Y cada tres años RX de tórax (PA y lateral).

Cuando en un reconocimiento médico postocupacional, se detecte alguno de los síntomas o signos descritos en el apartado anterior, se remitirá al especialista de neumología para estudio.

Por otra parte la OMS recomienda revisiones médicas periódicas a personas con antecedentes de exposición laboral a amianto. De esta forma se aconseja realizar radiografía de tórax cada 3-5 años a trabajadores con menos de 10 años desde la primera exposición; cada 1-2 años a trabajadores con más de 10 años desde la primera exposición y anualmente a trabajadores con más de 20 años desde la primera exposición. La frecuencia puede ser ajustada dependiendo de la edad del trabajador y de la intensidad y duración de la exposición. Asimismo se aconseja realizar cuestionario de síntomas respiratorios, exploración física y espirometría anual o de forma alternativa, coincidiendo con el 
estudio radiográfico. La vigilancia médica ha de realizarse de por vida?

La American Thoracic Society también recomienda monitorizar con radiografía de tórax y pruebas de función respiratoria cada 3-5 años a personas con antecedente de exposición laboral a amianto con más de 10 años desde la primera exposición.

En la actualidad existe consenso en que la prevención de la patología derivada del asbesto se basa en la eliminación de la exposición. Los exámenes de salud periódicos además de ejercer una función social pueden permitir actuar sobre factores como el tabaquismo que pueden agravar las patologías respiratorias ${ }^{8}$.

En el caso de la fibrosis pulmonar hay autores que sugieren que la enfermedad podría no evolucionar si se realiza interrupción precoz de la exposición al detectar los primeros signos de enfermedad ${ }^{9}$.

\section{BIBLIOGRAFÍA}

1. Boletín Oficial del Estado. Orden de 31/10/84 del Ministerio de Trabajo y Seguridad Social, Reglamento sobre Trabajos con riesgo de amianto. BOE núm. 267, 7/11/84.
2. Boletín Oficial del Estado. Orden de 26/7/93 del Ministerio de Trabajo y Seguridad Social, por la que modifica el Reglamento sobre Trabajos con riesgo de amianto y las normas complementarias. BOE núm. 188, 5/8/93.

3. Boletín Oficial del Estado. Orden 7/12/01 por la que modifica el anexo I del Real Decreto 1406/1989.

4. Villanueva V, Ballester R, Celma C, Ferris JM, Folch J, Fuster A et al. Protocolos de Vigilancia Sanitaria Específica. Amianto. Madrid: Ministerio de Sanidad y Consumo 1999

5. NELSON HN, Kelsey KT. The molecular epidemiology of asbestos and tabacco in lung cancer. Oncogene 2002; 21: 7284-7288.

6. Sociedad Española de Neumología y Cirugía Torácica. Normativa sobre el Asbesto y su patología pleuropulmonar 2004. Ed Doyma.

7. WAGNER GR. Asbestosis and silicosis. Lancet 1997; 349: 1311-1315.

8. American Thoracic Society. Diagnosis and Initial Management of Nonmalignant Diseases Related to Asbestos. Am J Respir Crit Care Med 2004; 170: 691-715.

9. SÉBASTIEN P, BÉGin R. Etiopatogenia de las Neumoconiosis. En: Stellman JM (dir). Enciclopedia de Salud y Seguridad en el Trabajo. Madrid: Ministerio de Trabajo y Asuntos Sociales, 2001. 